\title{
Efeitos de Exercícios Aquáticos e Orientação Nutricional na Composição Corporal de Crianças e Adolescentes Obesos
}

\author{
Effects of aquatic exercise and nutritional guidance on the body \\ composition of obese children and adolescents
}

\author{
Neiva Leite \\ Leilane Lazarotto \\ Jean Fuzetti Cavazza \\ Maria de Fátima Aguiar Lopes \\ Paulo Cesar Barauce Bento \\ Renata Torres \\ Maria Emilia D. Von der Heyde \\ Fabrício Cieslak \\ Gerusa Eisfeld Milano
}

1 Universidade Federal do Paraná. Departamento de Educação Física. Núcleo de Pesquisa em Qualidade de Vida. Curitiba, PR. Brasil.

2 Universidade Federal do Paraná. Departamento Nutrição. Núcleo de Pesquisa em Qualidade de Vida. Curitiba, PR. Brasil.

Recebido em 26/05/09 Revisado em 23/06/09 Aprovado em 02/12/09
Resumo - O objetivo do estudo foi analisar os efeitos de exercícios físicos aquáticos e orientação nutricional sobre a composição corporal em crianças e adolescentes obesos. Foram estudados 30 obesos com índice de massa corporal (IMC) acima do percentil 95, de acordo com o índice de massa corporal (IMC) proposto pela classificação de Center for Disease Control and Prevention (CDC, 2000), entre 10 e 16 anos, divididos em dois grupos: o primeiro grupo com iniciação e aprendizagem à natação (GNat, n=10) e o segundo com caminhada aquática em suspensão $(\mathrm{Gcam}=20)$. Na fase inicial e após 12 semanas, foram avaliados: peso, estatura, Índice de Massa Corporal (IMC), IMC-Escore Z, gordura relativa e absoluta, massa corporal magra relativa e absoluta. $O$ programa consistiu de 12 encontros para a orientação nutricional (uma vez por semana) e 36 sessões de exercício físico (três vezes por semana). Utilizaram-se MANOVA e Teste Exato Fischer para a análise estatística, com nível de significância $\mathrm{p}<0,05$. Após 12 semanas, o GCam apresentou redução significativa do IMC, IMC-Escore Z, gordura relativa e absoluta e aumento da massa corporal magra relativa e absoluta. Quando comparados os grupos, houve diferenças significativas para o GCam quanto à redução da gordura corporal $(\mathrm{p}=0,014)$ e aumento da massa magra ( $\mathrm{p}=$ 0,006) em relação ao GNat. O programa de caminhada aquática em suspensão aliado a um programa nutricional, pode resultar em importantes modificações na composição corporal de crianças e adolescentes obesos pela característica cíclica da atividade em relação ao grupo de aprendizado de natação.

Palavras-chave: Obesidade infanto-juvenil; Caminhada aquática em suspensão; Natação.

Abstract - The objective of this study was to analyze the effects of aquatic exercise and nutritional guidance on the body composition of obese children and adolescents. Thirty obese children aged 10 to 16 years, with a body mass index (BMI) above the 95th percentile according to the classification proposed by the Centers for Disease Control and Prevention (CDC, 2000), were divided into two groups: the first group started learning to swim (GSwim, $n=10$ ) and the second group underwent a deep water running program (GDeep, $n=20$ ). Weight, height, BMI, BMI $Z$-scores, relative and absolute fat, and relative and absolute lean body mass were evaluated at baseline and after 12 weeks. The program consisted of 12 meetings for nutritional guidance (once a week) and 36 sessions of exercise (three times per week). MANOVA and Fisher's exact test were used for statistical analysis, with the level of significance set at $p<0.05$. After 12 weeks, GDeep showed a significant reduction in BMI, BMI Z-scores and relative and absolute fat, and an increase in relative and absolute lean body mass. Significant differences were observed between GDeep and GSwim in terms of the reduction in body fat $(p=0.014)$ and the increase in lean mass $(p=0.006)$. A program of deep water running together with nutritional guidance may promote significant changes in the body composition of obese children and adolescents due to the cyclic characteristic of this physical activity.

Key words: Child obesity; Deep water running; Swimming. 


\section{INTRODUÇÃO}

A obesidade tornou-se um dos maiores problemas de saúde pública contemporânea e vem aumentando significativamente em todo o mundo, principalmente, em países desenvolvidos e em desenvolvimento. É caracterizada como uma doença crônica de comportamento epidêmico e multicausal ${ }^{1}$, estando associada ao desenvolvimento de outras morbidades e mortalidade 2 . O avanço da tecnologia é um dos contribuintes para que crianças e adolescentes tornem-se menos ativos, que em conjunto com a baixa qualidade e alta quantidade de alimentos proporciona maiores chances de desenvolver obesidade ${ }^{3,4}$.

A prática regular de exercícios físicos, em longo prazo, está associada com a atenuação da morbidade e risco de mortalidade em indivíduos obesos ${ }^{5}$. Contribui, também, com a melhora e manutenção da saúde, como alterações benéficas na capacidade cardiorrespiratória, e composição corporal ${ }^{6}$.

Exercícios aeróbios em terra oferecem muitos benefícios, no entanto, o praticante está sujeito a apresentar sintomas como dores, superaquecimento, transpiração e sensação de exaustão. A água permite que ocorram todos os benefícios dos exercícios em terra, com a vantagem de não apresentar efeitos colaterais $^{7-9}$. Atividades cíclicas, como a caminhada aquática em suspensão e a natação, auxiliam no controle e redução da gordura corporal e na melhora da aptidão cardiorrespiratória, pois proporcionam maior gasto energético, reduzem o impacto articular, devido à flutuação, e também problemas relacionados à temperatura corporal, facilitando a dissipação de calor ${ }^{7,8}$. A necessidade de oferecer um programa estimulador que promova gasto energético e que garanta segurança faz considerar a caminhada na água como alternativa de exercício físico para manejo da obesidade?.

$\mathrm{Na}$ caminhada aquática em suspensão, o praticante permanece em posição vertical, com o auxílio de um colete flutuador preso à cintura, de modo que o corpo fique submerso até a altura dos ombros, impossibilitando o contato dos pés com o fundo da piscina. Seu movimento é semelhante à corrida em terra, no entanto o aluno é submetido a um esforço muito intenso, devido às propriedades físicas da água. A aprendizagem depende de um curto período de familiarização, portanto, pode ser uma opção para o programa de exercícios destinado à pessoas obesas possibilitando o incremento progressivo de carga no decorrer do programa sem prejuízo das funções osteoarticulares ${ }^{10}$.

A adesão a uma proposta terapêutica é um dos maiores desafios enfrentados por profissionais da saúde. São observadas reduções na participação em programas com tratamentos longos, de natureza preventiva e quando há necessidade de mudança comportamenta ${ }^{11}$. A não adesão inicial auxilia na baixa evolução dos efeitos benéficos do exercício, entre eles, a redução da gordura corporal ${ }^{12,13}$.

Com relação à prática de atividades físicas regulares e educação nutricional, vários estudos nacionais e internacionais foram desenvolvidos, utilizando atividades terrestres ${ }^{3,14-17}$. Entretanto, poucos foram os estudos encontrados utilizando a caminhada aquática em suspensão ou natação ${ }^{18}$ como forma de tratamento em crianças e adolescentes obesos.

Tendo em vista a carência de estudos que envolvam atividades aquáticas e crianças e adolescentes obesos, o presente estudo objetivou avaliar os efeitos de dois diferentes exercícios físicos aquáticos e orientação nutricional sobre a composição corporal em crianças e adolescentes obesos.

\section{PROCEDIMENTOS METODOLÓGICOS}

\section{Desenho e sujeitos da pesquisa}

O delineamento do estudo é qualificado como quaseexperimental. A coleta de dados foi realizada no período de Junho de 2007 a dezembro de 2007, com crianças e adolescentes obesos. Foram avaliados 30 obesos, com idade entre 10 e 16 anos, sendo 11 do sexo feminino e 18 do sexo masculino, participantes de um programa de exercício físico e orientação nutricional.

O programa foi constituído por duas atividades aquáticas e os participantes foram divididos por conveniência nos dois grupos: caminhada aquática em suspensão (GCam ; n = 20) e iniciação e aprendizagem em natação (GNat; $\mathrm{n}=10$ ), realizada no Centro de Educação Física e Desporto (CED), no campus do Centro Politécnico da UFPR.

Os critérios de inclusão ao programa foram a classificação do Índice de Massa Corporal (IMC) igual ou superior ao $95^{\circ}$ percenti $1^{19}$ e participantes cujos responsáveis concordaram e assinaram o Termo de Consentimento Livre e Esclarecido (TCLE), conforme documento aprovado no Comitê de Ética do Setor de Saúde da UFPR, atendendo a resolução 196/96 do Conselho Nacional de Saúde sobre pesquisa, envolvendo seres humanos. Foram excluídos aqueles que utilizavam medicamentos que interferissem no controle do peso corporal.

\section{Avaliação Antropométrica e Clínica}

Todos participaram das avaliações no período inicial e após 12 semanas de tratamento. A avaliação clínica foi realizada pela mesma examinadora, 
profissional da área de pediatria, a fim de detectar possíveis doenças cardiovasculares. Nenhum indivíduo apresentou contra-indicação para a realização das atividades.

As técnicas utilizadas para obtenção das medidas antropométricas foram realizadas conforme Lohman $^{20}$. As medidas foram efetuadas pelo mesmo avaliador, com a realização de três medidas para consideração do valor médio entre as mesmas.

$\mathrm{Na}$ obtenção da estatura corporal $(\mathrm{cm})$, utilizou-se um estadiômetro (Ayrton Corporation, precisão de $0,1 \mathrm{~cm}$ ) fixado à parede, sendo que o indivíduo deveria manter-se em posição ortostática, com os pés unidos, descalços, utilizando o mínimo possível de roupas. Além disso, deveria permanecer em apnéia respiratória e com a cabeça no plano horizontal de Frankfort, tendo as superfícies posteriores do calcanhar, cintura pélvica, cintura escapular e região occipital em contato com o instrumento de medida.

A massa corporal $(\mathrm{kg})$ foi mensurada em balança (Filizola, precisão de 0,1 kg) tipo plataforma, com o avaliado permanecendo em posição ortostática, no centro da plataforma, descalço e utilizando roupas íntimas.

Em seguida, calculou-se o índice de massa corporal (IMC) mediante a utilização do índice de Quetelet, em $\mathrm{kg} / \mathrm{cm}^{2}$ : IMC=massa corporal/estatura $^{2}$. Para IMC, os indivíduos foram classificados segundo os critérios definidos pelo CDC (2000), conforme sexo, idade e etnia.

Para determinação da CA, foi utilizada uma fita antropométrica inelástica (precisão de 0,1 cm), aplicada no ponto médio entre a crista ilíaca e a face externa da última costela, paralela ao solo, com o sujeito em pé, com o abdômen relaxado, os braços ao longo do corpo e os pés unidos. A classificação da circunferência abdominal (CA) foi baseada nos critérios propostos por Fernande $z^{21}$, que considera os valores acima ou iguais ao $75^{\circ}$ percentil como limítrofes ou aumentados, conforme idade, sexo e etnia.

\section{Avaliação da Composição Corporal}

Para determinação da composição corporal, foi utilizado o método de impedância bioelétrica (BIA) tetrapolar, através do aparelho Maltron modelo BF906, com o posicionamento dos eletrodos nas superfícies dorsais das mãos e pés direitos, próximos às articulações metacarpofalangeanas e metatarso-falangeanas e medialmente entre as proeminências distais do rádio e da ulna e entre o maléolo tibial e fibular.

\section{Orientação Nutricional}

As atividades de orientação nutricional foram de- senvolvidas por uma nutricionista aluna do curso de pós-graduação Lato-Sensu de Nutrição Clínica da UFPR. Os encontros ocorreram uma vez por semana, com duração de uma hora. Durante o tratamento, foi realizado um inquérito alimentar validado para crianças e adolescentes, utilizando-se o registro de três dias ${ }^{22}$. Foram discutidas a aplicação prática da pirâmide alimentar, quanto à sua distribuição e porcionamento dos alimentos, a compreensão da leitura de rótulos, como melhor selecionar os alimentos e por fim, as possibilidades de mudanças na alimentação diária de cada participante.

\section{Prescrição de Exercícios Físicos Aquáticos}

As crianças e adolescentes que participaram do GCam passaram por um processo de adaptação ao meio líquido e familiarização ao gesto técnico da modalidade durante uma semana. As atividades foram desenvolvidas durante 12 semanas, sendo três sessões semanais com uma hora de duração, em uma piscina de 25 metros, com profundidade de 1,30 metros e temperatura média da água em $27,5^{\circ} \mathrm{C}$.

Para determinação da frequência cardíaca de treinamento (FCtreino), foi realizado teste de esforço máximo específico em piscina, simulando os movimentos da corrida, com auxílio de um colete flutuador. Para obter a frequência cardíaca de repouso (FCrep), em água, previamente ao início do teste, o avaliado permaneceu em repouso durante cinco minutos, em posição sentada dentro da piscina, com auxílio do aquatubo. $O$ protocolo para mensuração da frequência cardíaca máxima (FCmáx) consistiu em quatro repetições de 50 metros, com incremento de velocidade a cada repetição, caracterizadas por esforço leve, moderado, forte e máximo. Ao final do quarto estágio, o indivíduo que não atingiu a exaustão, realizou um quinto estágio. Em seguida, foi calculada a frequência cardíaca de reserva (FCres), subtraindo-se a frequência cardíaca de repouso da frequência cardíaca máxima.

A intensidade do exercício estabeleceu-se entre $35 \%$ a $55 \%$ da frequência cardíaca de reserva, durante a $1^{\mathrm{a}}$ e $4^{\mathrm{a}}$ semana, aumentando para $45 \%$ a $65 \%$, da $5^{\mathrm{a}}$ a $8^{\mathrm{a}}$ semana e atingindo entre $55 \%$ e $75 \%$, da $9^{a}$ a $12^{a}$ semana. A faixa de frequência cardíaca de treinamento foi determinada pela fórmula de frequência cardíaca de reserva, FCtreino=intensidadex (FCmáx - FCrep $)+$ FCrep .

Cada sessão de caminhada aquática em suspensão foi composta por 5 minutos de aquecimento, 45 minutos de atividade específica e 10 minutos de alongamento e recreação. A frequência cardíaca de treinamento pré-estabelecida da atividade física foi 
controlada durante a aula, através de frequencímetro da marca Polar ${ }^{\circledR}$ modelo F1.

O grupo natação foi dividido em iniciação e aprendizagem à natação, as aulas foram realizadas três vezes por semana, durante uma hora. Cada sessão foi dividida em três momentos: 5 minutos de aquecimento, 45 minutos de técnica e 10 minutos de alongamento e recreação.

\section{Análise Estatística}

Para análise descritiva das variáveis, foram utilizados os procedimentos de média, desvio-padrão e frequência, representados através de tabelas e gráficos. O Teste de Shapiro-Wilk foi utilizado para verificar a normalidade dos dados, em casos de ausência de normalidade, foram transformados para função de Logaritmo Natural ( $\operatorname{LogN})$. O Teste de Levene para verificar homogeneidade de variâncias, MANOVA para Medidas Repetidas e Teste Exato de FISHER para analisar as frequências $(\mathrm{p}<0,05)$. As análises foram realizadas através do programa estatístico Statistical Package for the Social Sciences (SPSS, versão 8.0) for Windows.

\section{RESULTADOS}

Participaram deste estudo 30 crianças e adolescentes obesos, com IMC igual ou superior ao $95^{\circ}$ percentil, de ambos os sexos, entre 10 e 16 anos. A amostra foi dividida em dois grupos, sendo 20 indivíduos (meninos $n=14$; meninas $n=6$ ) que realizaram a caminhada aquática em suspensão (GCam) e 10 (meninos $n$ $=5$; meninas $\mathrm{n}=5$ ) indivíduos que participaram da iniciação e aprendizagem à natação (GNat).

Entre os grupos GCam e GNat não houve diferenças significativas quanto às variáveis estudadas na fase inicial do estudo (Tabela 1).
Após 12 semanas de orientação nutricional e atividades físicas aquáticas, o GCam reduziu o IMC Escore-Z, gordura relativa e gordura absoluta. Os valores referentes à estatura, massa corporal relativa e absoluta apresentaram aumento ao final do tratamento no GCam . Por outro lado, ao analisar o grupo GNat, apenas a variável estatura aumentou ao final das 12 semanas. Quando comparados os grupos, após as 12 semanas foram encontradas diferenças significativas positivas para o grupo GCam em relação ao GNat, quanto à redução da gordura relativa e absoluta e aumento da massa corporal magra relativa e absoluta (Tabela 2).

O nível de adesão foi indicado através da permanência dos alunos nas atividades desenvolvidas, durante 12 semanas do programa de exercícios físicos aquáticos e orientação nutricional, considerando como boa adesão a participação mínima de duas vezes por semana, ou seja, frequência igual ou superior a $65 \%$ e baixa adesão uma frequência menor que $65 \%$. Após 12 semanas de atividades aquáticas e orientação nutricional, finalizaram 29 crianças e adolescentes obesos. A adesão do GCam foi de 90\% e do GNat $60 \%$, caracterizando maior adesão no primeiro grupo em comparação com o segundo ( $\mathrm{p}<0,05)$ (Figura 1).

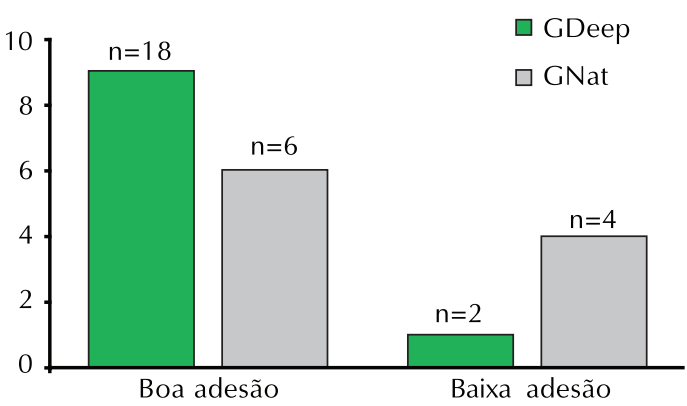

Figura 1. Nível de adesão após 12 semanas de intervenção Teste de Fischer=15,000; $<0,001$.

Tabela 1. - Médias e desvios-padrão das características gerais da amostra

\begin{tabular}{lcccc}
\hline Variável & GCam $(\mathrm{n}=19)$ & GNat $(\mathrm{n}=10)$ & $\mathrm{T}$ & valor de $\mathrm{p}$ \\
\hline Idade (anos) & $11,52 \pm 1,92$ & $12,9 \pm 2,99$ & $-1,579$ & 0,144 \\
Peso $(\mathrm{kg})$ & $73,97 \pm 26,75^{*}$ & $78,85 \pm 25,46^{*}$ & $-0,676$ & 0,544 \\
Estatura $(\mathrm{cm})$ & $155,49 \pm 10,63$ & $157,70 \pm 10,64$ & $-0,557$ & 0,600 \\
IMC $\left(\mathrm{kg} / \mathrm{m}^{2}\right)$ & $29,93 \pm 7,15^{*}$ & $30,00 \pm 7,91^{*}$ & $-0,545$ & 0,590 \\
IMC escore Z & $4,07 \pm 1,96^{*}$ & $3,86 \pm 1,53^{*}$ & 0,214 & 0,814 \\
CA (cm) & $97,34 \pm 13,37^{*}$ & $101,16 \pm 16,28^{*}$ & $-0,743$ & 0,502 \\
$\%$ Massa Gorda & $37,26 \pm 3,71$ & $36,5 \pm 8,26$ & 0,365 & 0,731 \\
Massa Gorda $(\mathrm{kg})$ & $27,68 \pm 11,10^{*}$ & $30,26 \pm 17,47^{*}$ & $-0,325$ & 0,806 \\
$\%$ Massa Magra & $62,73 \pm 3,71$ & $63,5 \pm 8,26$ & $-0,365$ & 0,731 \\
Massa Magra $(\mathrm{kg})$ & $45,75 \pm 14,33^{*}$ & $48,59 \pm 9,89^{*}$ & $-0,869$ & 0,425 \\
\hline
\end{tabular}

aDiferença significante para os dados iniciais.

*Dados que foram transformados para valores de logaritmo natural ( $\log N)$. 
Tabela 2. Características iniciais e finais da amostra

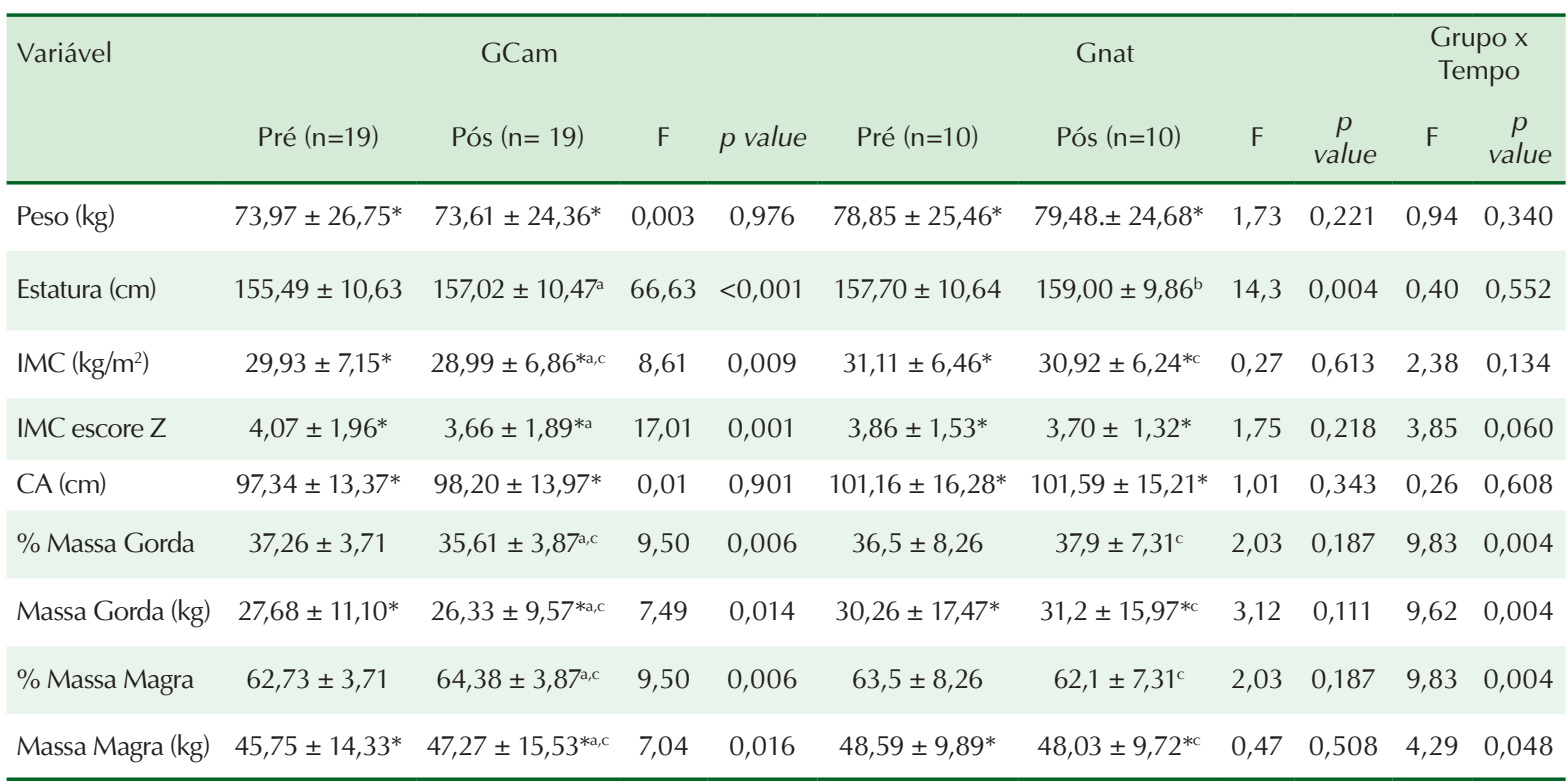

${ }^{a}$ Diferença significante de 12 semanas no grupo GCam .

${ }^{b}$ Diferença significante de 12 semanas no GNat.

'Diferença significante relativa à interação entre o GCam e o GNat.

*Dados que foram transformados para valores de logaritmo natural (LogN).

\section{DISCUSSÃO}

A primeira proposta deste estudo foi avaliar os efeitos de dois diferentes exercícios físicos aquáticos e orientação nutricional sobre a composição corporal em crianças e adolescentes obesos. Muitos estudos têm proposto a prática de atividade física como uma ferramenta essencial no combate à obesidade, entretanto, pouco tem se estudado o efeito de exercícios realizados na água como um instrumento atrativo para essa população ${ }^{3,14}$. Desta forma, na busca da prevenção e controle da obesidade é possível utilizar atividades aquáticas que podem facilitar a prática e consequentemente, aumentar a adesão, devido às propriedades físicas da água, como a viscosidade, a qual proporciona esforço intenso durante o exercício?.

Como as atividades na água podem ter um caráter mais lúdico, esse pode ser um ponto positivo para a adesão à atividade proposta. Segundo Robinson \& Rogers ${ }^{22}$, estima-se que cerca de $50 \%$ dos indivíduos que iniciam um programa de atividade física interrompem-no nos primeiros seis meses. Dentre os motivos de desistência estão o histórico da inatividade física, os baixos níveis de capacidade motora, a baixa tolerância ao exercício, falta de motivação pessoal, frustração após constatar objetivos difíceis de alcançar ${ }^{23}$. A adesão de obesos à atividade física varia de $50 \%$ a mais de $90 \%$ e está inversamente relacionada com a dura- ção dos programas ${ }^{24}$. Alguns estudos com crianças e adolescentes obesos que realizaram atividades fora da água, como caminhada e ciclismo indoor, encontraram uma adesão média de 80 a 86\%, em 12 semanas de atividade ${ }^{3,25}$. Entretanto, não foram encontrados trabalhos que avaliassem o nível de adesão de crianças e adolescentes obesos em atividades aquáticas. Neste estudo, com duração de 12 semanas, a adesão geral às atividades foi de 90\% no GCam e 60\% no GNat.

Apesar dos benefícios que os exercícios em água oferecem, é escasso o número de trabalhos que utilizam essa metodologia para o tratamento da obesidade. Desta forma, os poucos estudos com a prática da caminhada aquática em suspensão encontraram melhora da condição cardiorrespiratória e redução da gordura corporal em adultos obesos ${ }^{26-28}$. Contudo, no presente estudo, apesar das crianças e adolescentes do GCam não apresentarem uma redução no peso corporal, houve uma redução na massa gorda e um aumento na massa magra. Alguns trabalhos sugerem que na tentativa de minimizar a perda de peso, ao reduzir a massa livre de gordura, conseqüentemente também é reduzido o metabolismo basal, mesmo com a prática de exercícios físicos ${ }^{29}$. Dessa forma, o fato de a caminhada aquática em suspensão aumentar a massa magra e reduzir a massa gorda mostra-se como um fator relevante para o tratamento da obesidade em crianças e adolescentes.

Na natação, todos os participantes iniciaram o 
programa na fase de iniciação e aprendizagem, as quais são caracterizadas como atividades de baixo dispêndio energético, pois se trata de um período em que os exercícios são isolados, descontínuos e de curta duração, contrariando o caráter cíclico e contínuo de seus exercícios em fase avançada. Deste modo, na presente amostra, a iniciação à natação não proporcionou alterações nas variáveis antropométricas estudadas. Corroborando este estudo, outra pesquisa que utilizou a natação, assim como outras duas atividades (jogos aquáticos e futebol) como tratamento para a obesidade, durante 35 semanas, não encontrou alterações no peso corporal ${ }^{18}$.

O monitoramento da intensidade do GNat não foi realizado pelo motivo de que durante o período de iniciação e aprendizagem os exercícios são isolados e descontínuos, não possibilitando o controle adequado da intensidade, caracterizando a limitação deste estudo. Além disso, fazem-se necessários novos estudos com amostra e tempo de intervenção maior, com um grupo obeso que apresente uma técnica de natação mais avançada e um maior controle na prescrição de reeducação alimentar.

\section{CONCLUSÃO}

O tipo de modalidade, o nível de habilidade e familiarização ao gesto técnico pode influenciar no resultado do tratamento da obesidade. O programa de caminhada aquática em suspensão, quando realizado com frequência semanal adequada e intensidade de exercício monitorada, aliado a um programa nutricional, pode resultar em importantes modificações na composição corporal de crianças e adolescentes obesos pela característica cíclica das atividades e o processo de aprendizado do gesto motor ser rápido.

Os melhores resultados do GCam, provavelmente, estão relacionados à composição corporal e ocorreram por seu movimento assemelhar-se ao da corrida terrestre, portanto, seu gesto motor é de fácil e rápida assimilação, possibilitando movimentos cíclicos necessários para aumentar o gasto energético durante uma atividade aeróbia. Por outro lado, durante a fase de iniciação e aprendizagem à natação, os exercícios caracterizam-se por serem isolados e descontínuos, deixando de possuir caráter cíclico, portanto, de baixo gasto energético. Esses fatos também colaboram a maior adesão do GCam ao tratamento, pois a facilidade no aprendizado da atividade contribui para manter a motivação do participante. Sugerem-se novos estudos em crianças e adolescentes obesos, em programas com maior duração, controle da intensidade e uma amostra maior.

\section{REFERÊNCIA BIBLIOGRÁFICAS}

1. Oliveira AMA, Cerqueira EMM, Oliveira AC. Prevalência de sobrepeso e obesidade infantil na cidade de Feira de Santana-BA: detecção na família x diagnóstico clínico. J Pediatr 2003;79(4):325-28.

2. Steinbeck KS. The importance of physical activity in the prevention of overweight and obesity in childhood: a review and an opinion. Obes Rev 2001;2(2):117-30.

3. Leite N, Milano GE, Cieslak F, Lopes WA, Rodacki A, Radominski RB. Effects of physical exercise and nutritional guidance on metabolic syndrome in obese adolescents. Rev Bras Fisioter 2009;13(1):73-81.

4. Vitalle MSS, Tomioka CY, Juliano Y, Amancio, Olga MS. Índice de massa corporal, desenvolvimento puberal e sua relação com a menarca. Rev Assoc Med Bras 2003;49(4):429-33.

5. Francischi RPP, Pereira LO, Freitas CS, Klopfer M, Santos RC, Vieira P, et al. Obesidade: atualização sobre sua etiologia, morbidade e tratamento. Rev. Nutr 2000; 13(1):17-28.

6. Mcardle WD, Katch FI, Katch VL. Essentials of exercise physiology. 2 ed. Philadelphia: Lippincott, 2000.

7. Baum G. Aquaeróbica: manual de treinamento. 1 ed. São Paulo SP: Manole, 2000.

8. Owens SG. Obesity. In: Skinner JS. Exercise Testing and Exercise Prescription for Special Cases: Theoretical Basis and Clinical Application. 3 ed. New York: Lippincott Williams \& Wilkins, 2005, p. 237-53.

9. Lazzari JMA, Meyer F. Freqüência cardíaca e percepção de esforço na caminhada aquática e na esteira em mulheres sedentárias e com diferentes pecentuais de gordura. Rev Bras Ativ Física Saúde 1997;2(3):7-13.

10. Delgado CA, Delgado SN. A prática da hidroginástica. Rio de Janeiro: Sprint, 2001.

11. Malerbi FEK. Adesão ao tratamento. In: Kerbauy RR. Sobre comportamento e cognição. Vol 5. Santo André: Editora SET, 2000, p. 148-55.

12. Fechio JJ, Malerbi FEK. Adesão de um programa de atividade física em adultos portadores de diabetes. Arq Bras Endocrinol Metab 2004; 48(4):267-75.

13. Vieira MA, Atihê RD, Soares RC, Damiani D, Setian N. Adesão inicial ao tratamento de adolescentes obesos em programas de redução de peso. Pediatria 2004; 26(4):240-6.

14. Caranti DA, Mello, MT, Prado, WL, Tock L, Siqueira KO, Piano A, et al. Short- and long-term beneficial effects of a multidisciplinary therapy for the control of metabolic syndrome in obese adolescents. Metabolism 2007; 56(9):1293-300.

15. Nemet D, Barkan S, Epstein Y, Friedland O, Kowen G, Eliakim A. Short- and long-term beneficial effects of a combined dietary-behavioral-physical activity intervention for the treatment of childhood obesity. Pediatrics 2005;115(4):443-9. 
16. Parente EB, Guazzelli I, Ribeiro MM, Silva AG, Halpern A, Villares SM. Perfil lipídico em crianças obesas: efeitos de dieta hipocalórica e atividade física aeróbica. Arq Bras Endocrinol Metab 2006; 50(3):449-504.

17. Park TG, Hong HR, Lee J, Kang HS. Lifestyle plus exercise intervention improves metabolic syndrome markers without change in adiponectin in obese girls. Ann Nutr Metab 2007; 51(3):197-203.

18. Ildikó V, Zsófia M, János M, Andreas P, Dora NE, András P, Ágnes S, Zsolt S, Kumagai S. ActivityRelated Changes of Body Fat and Motor Performance in Obese Seven-Year-Old Boys. J Physiol Anthropol 2007; 26(3):333-7.

19. Kuezmarski RJ, Ogden CL, Grummer-Estrawn LM, Flegal KM, Guo SS, Wei R, et al. CDC growth charts: United States. Adv Data 2000:1-27.

20. Lohman TG, Roche AF, Martorell R. Anthropometric standardization reference manual. Champaign: Human Kinetics, 1988.

21. Fernandez JR, Redden DT, Petrobelli A, Allisson DB. Waist circumference percentiles in nationally representative samples of african-american, european-american, and mexican-american, children and adolescents. J Pediatr, 2004;145(4):439-44.

22. Slater B, Fisberg RM, Philippi ST, Latorre MRO. Validation of a semi-quantitative adolescents food frequency questionnaire applied at a public school in São Paulo, Brazil. Eur J Clin Nutr 2003; 57(5):629-35.

23. Robinson JI, Rogers MA. Adherence to exercise programmes: recommendations. Sports Méd 1994;17(1):39-52.

24. Nunomura M, Andreotti RA, Teixeira LA, Okuma SS. Nível de adesão ao exercício num programa supervisionado. Rev Bras Ativ Fís Saúde 1997;2(3):61-6
25. Coutinho W. Consenso Latino Americano sobre Obesidade. Arq Bras Endocrinol Metab 1999;43:21-67,

26. Rezende, BA. Nível de Adesão de crianças e adolescentes obesos em programa de condicionamento físico. [Trabalho de Conclusão de Curso-Graduação em Educação Física]. Curitiba (PR): Universidade Federal do Paraná, 2004.

27. Pasetti SR, Gonçalves A, Padovani CR. Corrida em piscina profunda para melhora da aptidão física de mulheres obesas na meia idade: estudo experimental de grupo único. Rev Bras Educ Fís Esp 2006;20(4):297-304.

28. Sherman NW, Michaud TJ. Aquarunning for improving muscular strength, endurance and flexibility? (Abstract). Med Sci Sports Exerc 1999; 31(5):312.

29. Swank SA, Long K, Lee E, Poindexter HB. Strength, flexibility and body composition changes of older women following 10 weeks of water exercise (Abstract). Med Sci Sports Exerc 1996;28(5):189.

30. Aliga MJ, Santos JL, Martínez JA. Does weight loss prognosis depend on genetic make-up? Obes Rev 2005;6:155-168.

\section{Endereço para correspondência}

Neiva Leite

Departamento de Educação Física, Universidade Federal do Paraná.

Rua Coração de Maria, 92 (BR-116, km 92).

Jardim Botânico

CEP 80215-370 - Curitiba. PR. Brasil

E-mail: neivaleite@gmail.com 